\title{
A phase II study of combination therapy with oral S-1 and cisplatin in elderly patients with advanced gastric cancer
}

\author{
Yusuke Sasaki $^{1} \cdot$ Satoru Iwasa $^{1} \cdot$ Shunsuke Okazaki $^{2} \cdot$ Masahiro Goto $^{3} \cdot$ Yasushi Kojima $^{4} \cdot$ Atsushi Naganuma $^{5}$. \\ Kengo Nagashima $^{6} \cdot$ Yushi Nagai $^{7} \cdot$ Hidekazu Hirano $^{1} \cdot$ Yoshitaka Honma $^{1} \cdot$ Atsuo Takashima $^{1} \cdot$ Ken Kato $^{1}$. \\ Tetsuya Hamaguchi ${ }^{1}$
}

Received: 10 April 2017 / Accepted: 17 July 2017 / Published online: 1 August 2017

(c) The International Gastric Cancer Association and The Japanese Gastric Cancer Association 2017

\begin{abstract}
Background A combination of S-1 and cisplatin is recognized as one of the standard first-line chemotherapy regimens for patients with advanced gastric cancer. However, demographic analyses of pivotal phase III studies have showed that only a minority of treated patients were aged 76 years or older. The purpose of this phase II study was to evaluate the safety and efficacy of combination therapy with S-1 and cisplatin in elderly patients with chemotherapynaive advanced gastric cancer.

Methods Patients aged 76 years or older received S-1 $40 \mathrm{mg} / \mathrm{m}^{2}$ orally twice daily for 21 days and cisplatin $60 \mathrm{mg} /$ $\mathrm{m}^{2}$ intravenously infused at day 8 of each 35 -day cycle. Dose modification was performed according to creatinine clearance. The primary endpoint was overall survival (OS). Secondary endpoints included response rate, progression-free
\end{abstract}

Satoru Iwasa

siwasa@ncc.go.jp

1 Gastrointestinal Medical Oncology Division, National Cancer Center Hospital, 5-1-1 Tsukiji, Chuo-ku, Tokyo 104-0045, Japan

2 Medical Oncology Division, National Hospital Organization Kyoto Medical Center, Kyoto, Japan

3 Cancer Chemotherapy Center, Osaka Medical College Hospital, Osaka, Japan

4 Department of Gastroenterology, National Center for Global Health and Medicine, Tokyo, Japan

5 Department of Gastroenterology, National Hospital Organization Takasaki General Medical Center, Gunma, Japan

6 Department of Global Clinical Research, Chiba University Graduate School of Medicine, Chiba, Japan

7 Clinical Trial Support Office, National Cancer Center Hospital, Tokyo, Japan survival (PFS), time to treatment failure (TTF), and adverse events.

Results A total of 40 patients were enrolled. Median OS was 12.3 months, PFS was 7.8 months, and TTF was 4.3 months. The response rate was $54 \%$. The most common grade 3-4 adverse events were anorexia (25\%), neutropenia (23\%), hyponatremia (20\%), anemia (18\%), and febrile neutropenia (8\%). No treatment-related death occurred.

Conclusions Combination chemotherapy with S-1 and cisplatin is an effective and well-tolerated regimen for elderly patients with advanced gastric cancer when the dose is adjusted according to renal function.

Keywords Cisplatin - Elderly patient - Gastric cancer . S-1

\section{Introduction}

Gastric cancer remains one of the most common forms of cancer worldwide, with an incidence of approximately 950,000 new cases and 720,000 deaths per year, accounting for approximately $6.8 \%$ of all new cancers [1]. In Japan, the incidence of gastric cancer was estimated at 29.9 per 100,000 new cases in 2012, giving Japan the third highest incidence in the world [1]. In the past few decades, despite a plateau in disease prevalence, an increasing incidence of gastric cancer in the elderly has been observed because of the increasing longevity of the general population [2].

Although some patients have gastric cancer that extends within locoregional confines and is amenable to curative resection, many patients are not candidates for curative resection at the time of diagnosis. Chemotherapy is the most effective treatment for patients with unresectable advanced and metastatic gastric cancer [3-5]. Combination therapy with a 
fluoropyrimidine and platinum drugs has been one of the most commonly used regimens because of its therapeutic activity and acceptable toxicity profile.

$\mathrm{S}-1$ is an oral fluoropyrimidine derivative that combines tegafur and two 5-fluorouracil (5-FU) modulators: gimeracil (5-chloro-2, 4-dihydroxypyridine; CDHP) and potassium oxonate (oteracil) in a molar ratio of 1:0.4:1. Tegafur, an oral prodrug of 5-FU, is gradually converted to 5-FU and rapidly metabolized by dihydropyrimidine dehydrogenase (DPD) in the liver. CDHP augments the activity of 5-FU by inhibiting DPD. Oteracil inhibits pyrimidine phosphoribosyl transferase specifically in the gastrointestinal tract, thereby reducing intestinal phosphorylation of 5-FU.

The comparable efficacy of regimens substituting S-1 for infused 5-FU has been directly studied in two phase III studies: the JCOG9912 study and the FLAGS study [6, 7]. Additionally, the SPIRITS study, comparing S-1 monotherapy with S-1 and cisplatin, concluded that the combination of S-1 and cisplatin showed a higher response rate and better overall survival (OS) than S-1 monotherapy [8]. The combination of S-1 and cisplatin is therefore recognized as one of the standard first-line chemotherapy regimens for patients in Japan with advanced gastric cancer. However, demographic analyses of phase III studies showed that only a minority of treated patients were aged 75 years or older. Elderly patients often present with concomitant co-morbidities and age-associated physiological problems, such as impaired organ function and functional changes that complicate the selection of optimal treatment. Furthermore, published data are limited concerning gastric cancer treatment in this specific age group for reasons of strict exclusion criteria and the consequent underrepresentation of elderly patients in clinical studies.

In previous clinical studies for elderly patients with gastric cancer, S-1 monotherapy achieved response rates of $21-29 \%$ and a median OS of 8-15 months $[9,10]$. However, there were no data about the impact of adding cisplatin to S-1 for the treatment of elderly patients with gastric cancer in a prospective study.

Thus, we investigated the safety and efficacy of combination therapy with S-1 and cisplatin in elderly patients with chemotherapy-naive advanced gastric cancer. Because both S-1 and cisplatin are normally eliminated by renal excretion, a dose reduction of these drugs is required in patients with renal impairment [11]. Therefore, we adjusted the dosage of $\mathrm{S}-1$ and cisplatin according to creatinine clearance $(\mathrm{CCr})$.

\section{Patients and methods}

\section{Patients}

Patients 76 years or older with histologically confirmed adenocarcinoma of the stomach or gastroesophageal junction were enrolled for this study. Before enrollment, patients had to fulfill all the following criteria: (i) histologically proven gastric adenocarcinoma; (ii) unresectable or recurrent disease; (iii) Eastern Cooperative Oncology Group (ECOG) performance status (PS) of 0 or 1; (iv) no prior hormone therapy, chemotherapy, or radiotherapy, although hormone therapy and adjuvant chemotherapy with fluoropyrimidine completed more than 24 weeks before entry were allowed; (v) adequate food intake; (vi) negative human epidermal growth factor receptor 2 (HER2) status (if the main histological type was diffuse, unknown status was allowed); and (vii) written informed consent. Patients were also required to have adequate hematological and organ function, defined by an absolute neutrophil count $\geq 1,500 / \mu 1$, platelets $\geq 100,000 / \mu$ l, total bilirubin less than $1.5 \mathrm{mg} / \mathrm{dl}$, aspartate transaminase and alanine aminotransferase less than $100 \mathrm{IU} / \mathrm{l}$, an estimated creatinine clearance $(\mathrm{CCr})$ of more than $30 \mathrm{ml} / \mathrm{min}$, and a creatinine level less than $1.5 \mathrm{mg} / \mathrm{dl}$. Patients were excluded for any of the following criteria: (i) synchronous or metachronous (within 5 years) malignancies other than carcinoma in situ or mucosal carcinoma; (ii) current treatment with systemic steroids; (iii) uncontrolled diabetes mellitus; (iv) history of myocardial infarction or unstable angina pectoris within 6 months; (v) active bleeding; or (vi) massive ascites or pleural effusion.

\section{Study design and treatment}

This was a multi-institutional, phase II study, in which participating institutions comprised 18 centers as of November 2012. This study was approved by the institutional review board at each participating center and conducted in accordance with the principles of the Declaration of Helsinki.

Patients received oral S-1 $40 \mathrm{mg} / \mathrm{m}^{2}$ twice daily for 21 days followed by 14 days of rest, and cisplatin $60 \mathrm{mg} /$ $\mathrm{m}^{2}$ intravenously infused on day 8 of each 35 -day cycle. $\mathrm{CCr}$ was calculated using the Cockcroft equation before each cycle of chemotherapy, and dose modifications were allowed according to the estimated $\mathrm{CCr}$ (Table 1). If the $\mathrm{CCr}$ before each cycle was reduced under the treatment, a dose reduction was necessary from that cycle. This dose-reduction criterion was decided by using a previous study and review as references $[10,12,13]$.

The protocol treatment was repeated for up to eight cycles. Patients continued protocol treatment cycles until disease progression or intolerable toxicity or they withdrew their consent from the study. S-1 monotherapy was recommended as a subsequent chemotherapy after eight cycles of the protocol treatment.

The study was registered with the University Hospital Medical Information Network (UMIN) Clinical Trials 
Table 1 Adjusted chemotherapy dose according to creatinine clearance

\begin{tabular}{|c|c|c|c|c|c|c|c|c|c|}
\hline \multirow{3}{*}{$\begin{array}{l}\text { BSA } \\
\mathrm{CCr}(\mathrm{ml} / \mathrm{min})\end{array}$} & \multicolumn{6}{|c|}{ Dose of S-1 (mg/day) } & \multicolumn{3}{|c|}{ Dose of cisplatin $\left(\mathrm{mg} / \mathrm{m}^{2}\right)$} \\
\hline & \multicolumn{2}{|c|}{$\mathrm{BSA}<1.25 \mathrm{~m}^{2}$} & \multicolumn{2}{|c|}{$1.25 \mathrm{~m}^{2} \leq \mathrm{BSA}<1.5 \mathrm{~m}^{2}$} & \multicolumn{2}{|c|}{$\mathrm{BSA} \geq 1.5 \mathrm{~m}^{2}$} & \multirow[t]{2}{*}{$\mathrm{CCr} \geq 60$} & \multirow[t]{2}{*}{$50 \leq \mathrm{CCr}<60$} & \multirow[t]{2}{*}{$30 \leq \mathrm{CCr}<50$} \\
\hline & $\mathrm{CCr} \geq 50$ & $30 \leq \mathrm{CCr}<50$ & $\mathrm{CCr} \geq 50$ & $30 \leq \mathrm{CCr}<50$ & $\mathrm{CCr} \geq 50$ & $30 \leq \mathrm{CCr}<50$ & & & \\
\hline Level 0 & 80 & 50 & 100 & 80 & 120 & 100 & 60 & 40 & 30 \\
\hline Level 1 & 50 & 40 & 80 & 50 & 100 & 80 & 40 & 30 & - \\
\hline Level 2 & 40 & - & 50 & 40 & 80 & 50 & 30 & - & - \\
\hline
\end{tabular}

$B S A$ body surface area, $C C r$ creatinine clearance calculated by Cockcroft equation

Registry (protocol ID: UMIN000009349) on 19 November 2012.

\section{Outcome measures and statistical methods}

The aim of this study was to evaluate the efficacy and safety of combination therapy with $\mathrm{S}-1$ plus cisplatin in elderly patients with advanced gastric cancer. The primary endpoint was OS. Secondary endpoints included an objective response rate, progression-free survival (PFS), time to treatment failure (TTF), and adverse events. The OS was defined as the time from the date of enrollment to death from any cause or last follow-up. The PFS was defined as the time from the date of enrollment to progression or death from any cause, and the TTF was defined as progression, death, or discontinuation of protocol treatment. Median TTF, PFS, and OS were estimated using the Kaplan-Meier method. For patient characteristics and adverse events, summary statistics were constructed using frequencies and/or proportions. The ratio of the actual dose taken to the prescribed dose was calculated and used to calculate relative dose intensity.

The sample size of the study was calculated as 36 patients with $80 \%$ power under the hypothesis that the expected value of the median OS was 14 months and the threshold value was 8 months using a Brookmeyer-Crowley type test at a one-sided significance level of 0.10 [14]. Assuming a dropout rate of $10 \%$, the required sample size was estimated to be 40 patients.

All statistical comparisons were planned before the study began. All statistical analyses were done using SAS Version 9.4 (SAS Institute, Cary, NC, USA).

\section{Follow-up}

Tumor assessments were performed at baseline and every 10 weeks until disease progression, which was based on computed tomography and/or magnetic resonance imaging evaluation as defined by Response Evaluation Criteria in Solid Tumors guidelines. Adverse events were also evaluated at baseline, at the beginning of each treatment cycle, and at day 8 using Common Terminology Criteria for Adverse Events (version 4.0).

\section{Results}

\section{Patient characteristics}

A total of 40 patients were enrolled at 15 institutions between November 2012 and October 2014. Patient and tumor characteristics are listed in Table 2 . The median age of patients was 78 years (range, 76-89 years); 76-80 years for 32 patients $(80 \%), 81-85$ years for $6(15 \%)$, and 86-89 years for 2 patients $0(5 \%)$. Twenty-nine patients (73\%) required an initial dose reduction of $\mathrm{S}-1$ or cisplatin according to the $\mathrm{CCr}$ before treatment; $30 \leq \mathrm{CCr}<50$ for 18 patients $(45 \%)$, and $50 \leq \mathrm{CCr}<60$ for $11(27 \%)$. Twenty-eight patients $(70 \%)$ had target lesions. Major co-morbidities were hypertension (38\%), hyperlipidemia (18\%), and diabetes (13\%).

\section{Study treatment}

A total of 150 cycles of treatment were given to patients; the median number of cycles for each patient was 4 (range, $1-8)$. Three patients ( $8 \%$ ) completed the 8 cycles of the protocol treatment. The relative dose intensity was $91.2 \%$ for S-1 and $91.5 \%$ for cisplatin of all treatment cycles prescribed in the protocol. Cisplatin was not given in $2.0 \%$ of all treatment cycles. A dose reduction of S-1 and cisplatin was needed for $30 \%$ and $35 \%$ of patients, respectively. The most common reasons for dose reduction were neutropenia and fatigue. No patients required a dose reduction according to a change of $\mathrm{CCr}$ under treatment. Although grade 3 increased serum creatinine was found in 1 patient, this patient discontinued treatment for reasons of severe anorexia and nausea in the middle of the first cycle. Treatment was discontinued in 17 patients (43\%) for disease progression, in 10 patients (25\%) for adverse events, and in 7 patients (17\%) for withdrawal of consent. Adverse events that led to a discontinuation were anorexia, neutropenia 
Table 2 Patient and tumor characteristics

\begin{tabular}{|c|c|}
\hline Parameter & $n(\%)$ \\
\hline \multicolumn{2}{|l|}{ Gender } \\
\hline Male & $29(73 \%)$ \\
\hline Female & $11(27 \%)$ \\
\hline \multicolumn{2}{|l|}{ Age } \\
\hline Median (range) & $78(76-89)$ \\
\hline \multicolumn{2}{|l|}{ PS (ECOG) } \\
\hline 0 & $13(32 \%)$ \\
\hline 1 & $27(68 \%)$ \\
\hline \multicolumn{2}{|l|}{ Diagnosis status } \\
\hline Metastatic & $30(75 \%)$ \\
\hline Recurrence & $10(25 \%)$ \\
\hline \multicolumn{2}{|c|}{ Histological main type } \\
\hline Intestinal & $20(50 \%)$ \\
\hline Diffuse & $19(48 \%)$ \\
\hline Mucinous & $1(2 \%)$ \\
\hline \multicolumn{2}{|l|}{ Site of metastasis } \\
\hline Liver & $11(28 \%)$ \\
\hline Lung & $3(8 \%)$ \\
\hline Lymph node & $33(83 \%)$ \\
\hline Peritoneal & $15(38 \%)$ \\
\hline \multicolumn{2}{|c|}{ Number of metastatic sites } \\
\hline 1 & $14(35 \%)$ \\
\hline 2 & $23(57 \%)$ \\
\hline 3 & $3(8 \%)$ \\
\hline \multicolumn{2}{|l|}{$\mathrm{CCr}(\mathrm{ml} / \mathrm{min})$} \\
\hline $30 \leq \mathrm{CCr}<50$ & $18(45 \%)$ \\
\hline $50 \leq \mathrm{CCr}<60$ & $11(27 \%)$ \\
\hline $60 \leq \mathrm{CCr}$ & $11(27 \%)$ \\
\hline
\end{tabular}

$P S$ performance status, ECOG Eastern Cooperative Oncology Group, $\mathrm{CCr}$ creatinine clearance calculated by Cockcroft equation

( 3 cases each), fatigue, and cognitive dysfunction ( 2 cases each).

\section{Efficacy}

After a median follow-up period of 11.8 months (range, 0.7-37.7 months), 30 patients had died ( 29 were cancer-related deaths and 1 was from a pulmonary thromboembolism). Median OS was 12.3 months ( $80 \%$ confidence interval, 10.2-14.4) (Fig. 1a). Median PFS was 7.8 months and median TTF was 4.3 months (Fig. 1b, c). Of the 28 patients who had target lesions, complete response (CR) was achieved by 1 patient and partial response (PR) by 14 patients, resulting in a response rate of $54 \%$. Second-line treatment was given to 20 patients (50\%; paclitaxel in $11, \mathrm{~S}-1$ alone in 5, irinotecan in 2, and radical surgery for tumor shrinkage in 2).

\section{Safety}

Table 3 shows hematological and nonhematological adverse events. The main adverse events at grade 3 or higher level were anorexia (25\%), neutropenia (23\%), hyponatremia (20\%), anemia (18\%), and febrile neutropenia (8\%). No treatment-related death occurred.

\section{Discussion}

This phase II study showed that combination chemotherapy with S-1 and cisplatin is effective and well tolerated in patients with advanced gastric cancer aged 76 years or older when the dose is adjusted depending on renal function.

In general, the incidence of elderly patients with gastric cancer is gradually increasing. The majority of patients show advanced disease when they are diagnosed, and systemic chemotherapy is considered the choice for palliative treatment. However, the elderly are less likely to receive chemotherapy because of major organ dysfunctions, concomitant diseases, and a shorter life expectancy, all of which lead to increased side effects of chemotherapy and reduced tolerance to treatment. Therefore, we investigated a phase II trial to evaluate the efficacy and safety of S-1 and cisplatin chemotherapy, taking into account patient renal function.

Several previous phase II studies on elderly patients with gastric cancer have shown that S-1 monotherapy achieves high response rates with low frequencies of serious adverse events. Lee et al. carried out a randomized phase II study in elderly patients aged 65 years or more, and reported that S-1 monotherapy yielded a response rate of $28.9 \%$, a median time to progression of 4.2 months, and a median OS of 8.2 months [9]. Koizumi et al. reported on a phase II study in elderly patients aged 75 years or more where S-1 monotherapy, adjusted according to the $\mathrm{CCr}$, provided a response rate of $21.2 \%$, a median TTF of 3.2 months, a median PFS of 3.9 months, and a median OS of 15.7 months [10]. In comparison with these studies, our study achieved better results in terms of response rate and survival. However, our study was a nonrandomized study and a bias in patient selection may have occurred. Therefore, it may be difficult to compare such results considering the greater heterogeneity in physical and psychosocial backgrounds in elderly patients.

As a phase III study of S-1 and cisplatin combination chemotherapy in patients 75 years of age or younger with advanced or recurrent gastric cancer, the SPIRITS trial yielded a response rate of 54\%, a median PFS of 6.0 months, and a median OS of 13.0 months [8]. In the SPIRITS study, a median four cycles of chemotherapy were given for each patient. The median relative dose intensity of S-1 was $93.3 \%$, and cisplatin was not given in $9.8 \%$ of all cycles of treatment. 

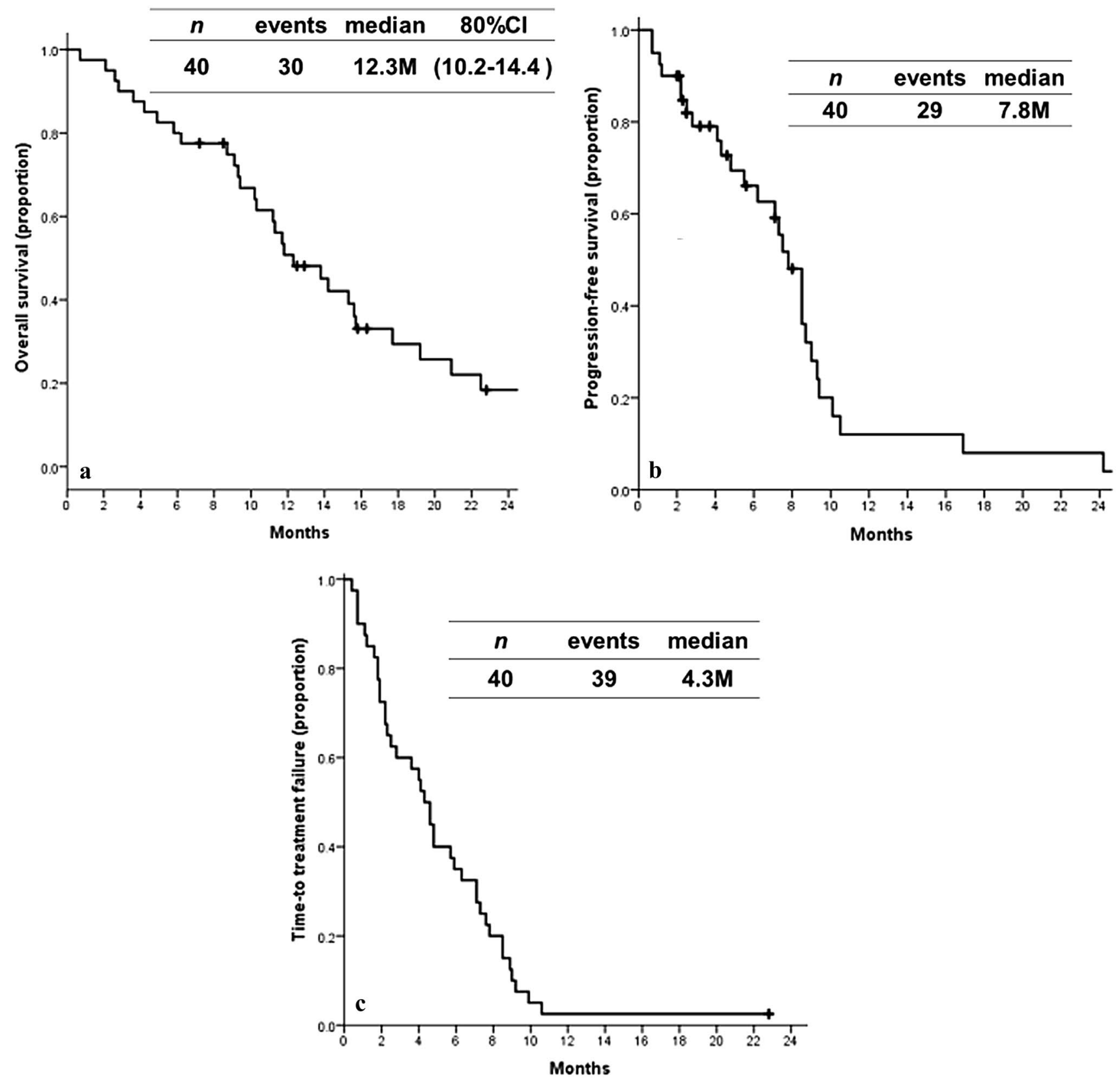

Fig. 1 Overall survival (a), progression-free survival (b), and time to treatment failure (c) in elderly patients with gastric cancer treated with oral S-1 and cisplatin. $C I$ confidence interval, $M$ months

Major grade 3 or 4 adverse events were neutropenia (40\%), anorexia (30\%), anemia (26\%), nausea (11\%), and febrile neutropenia (3\%). These results were not very different from the results of our study, although we noted more frequent febrile neutropenia (8\%). Additionally, in the SPIRITS study, the reasons for discontinuation of S-1 and cisplatin therapy were progressive disease (44\%), adverse events (28\%), or consent withdrawal (9\%). The proportion of adverse events was the same as our study, even though almost half the patients in our study were found to have renal dysfunction, with a $\mathrm{CCr}<50$. Additionally, the proportion of patients who withdrew consent was greater in our study. But, among the seven patients who withdrew consent in our study, five patients received four or more cycles of chemotherapy and severe adverse events were not found. Therefore, we consider that the preventative treatment in our trial, in which the dose was adjusted according to the $\mathrm{CCr}$, was as well tolerated in elderly patients as it was in younger patients. However, the presence of several adverse 
Table 3 Adverse events $(n=40)$

\begin{tabular}{lrc}
\hline & Any grade $(\%)$ & Grades \\
& & $3-4(\%)$ \\
\hline Any adverse event & 100 & 70 \\
Neutropenia & 60 & 23 \\
Febrile neutropenia & 8 & 8 \\
Anemia & 78 & 18 \\
Thrombocytopenia & 78 & 10 \\
Increased AST & 30 & 5 \\
Increased ALT & 30 & 8 \\
Increased creatinine & 18 & 3 \\
Hyponatremia & 70 & 20 \\
Anorexia & 75 & 25 \\
Nausea & 43 & 10 \\
Fatigue & 75 & 8 \\
Vomiting & 18 & 0 \\
Diarrhea & 40 & 5 \\
Stomatitis & 33 & 10 \\
Sensory neuropathy & 10 & 0 \\
Hand-foot syndrome & 13 & 0 \\
\hline
\end{tabular}

$A S T$ aspartate transaminase, $A L T$ alanine aminotransferase

events, such as cognitive dysfunction that is specific to the elderly, must be taken into account.

Oxaliplatin is a newer-generation platinum compound that improves tolerability when compared to cisplatin. Several phase III studies showed that oxaliplatin had a similar effect to cisplatin in young and elderly patients with previously untreated advanced gastric cancer [15-17]. Additionally, Santini et al. carried out a phase II study in Italian elderly gastric cancer patients. Weekly oxaliplatin, 5-FU, and leucovorin (OXALF) therapy provided a response rate of $45 \%$, a median time to progression of 5.0 months, and a median OS of 9.0 months [18]. The patients in this study were 70 years and older, of whom 33\% showed neurotoxicity. Sensory symptoms deteriorated over time and worsened the quality of life of elderly patients. In our study, grade 3 or higher sensory neuropathy was not observed.

This study had several limitations. Most notably, this investigation was a single-arm study performed in a limited number of patients. Additionally, the patients' quality of life, which may have become impaired as the intensity of chemotherapy increased, was not sufficiently studied.

In conclusion, the primary endpoint of this study was met: our results suggested that combination chemotherapy with S-1 and cisplatin is an effective and well-tolerated regimen for elderly patients with advanced gastric cancer when the dose is adjusted according to changes in renal function. This regimen may be considered an alternative treatment option in elderly patients with advanced gastric cancer.
Acknowledgements We are grateful to the participating patients and their families. We thank the Clinical Trial Support Office at the National Cancer Center Hospital for their assistance. We thank all SPEL study investigators.

Author contributions Data extraction was performed by Yusuke Sasaki and Yushi Nagai. Statistical analysis was performed by Kengo Nagashima. The manuscript was written by Yusuke Sasaki. All authors gave final approval for submission of the manuscript.

\section{Compliance with ethical standards}

Conflict of interest All authors declare that they have no conflicts of interest.

Ethical standards All procedures followed were in accordance with the ethical standards of the responsible committee on human experimentation (institutional and national) and with the Helsinki Declaration of 1964 and later versions. Informed consent or substitute for it was obtained from all patients for being included in the study.

Funding This study was supported by the Japan Society of Clinical Oncology.

\section{References}

1. GLOBOCAN database: http://globocan.iarc.fr/old/FactSheets/ cancers/stomach-new.asp. Accessed 02 May 2017.

2. Center for Cancer Control and Information Services, National Cancer Center, Japan: http://ganjoho.jp/public/cancer/stomach/. Accessed 02 May 2017.

3. Murad AM, Santiago FF, Petroianu A, Rocha PR, Rodrigues MA, Rausch M. Modified therapy with 5-fluorouracil, doxorubicin, and methotrexate in advanced gastric cancer. Cancer (Phila). 1993;72:37-41.

4. Glimelius B, Hoffman K, Haglund U, Nyrén O, Sjödén PO. Initial or delayed chemotherapy with best supportive care in advanced gastric cancer. Ann Oncol. 1994;5:189-90.

5. Pyrhönen S, Kuitunen T, Nyandoto P, Kouri M. Randomised comparison of fluorouracil, epidoxorubicin and methotrexate (FEMTX) plus supportive care with alone in patients with nonresectable gastric cancer. Br J Cancer. 1995;71:587-91.

6. Boku N, Yamamoto S, Fukuda H, Shirao K, Doi T, Sawaki A, et al. Fluorouracil versus combination of irinotecan plus cisplatin versus S-1 in metastatic gastric cancer: a randomised phase 3 study. Lancet Oncol. 2009;10:1063-9.

7. Ajani JA, Rodriguez W, Bodoky G, Moiseyenko V, Lichinitser $\mathrm{M}$, Gorbunova V, et al. Multicenter phase III comparison of cisplatin/S-1 with cisplatin/infusional fluorouracil in advanced gastric or gastroesophageal adenocarcinoma study: the FLAGS trial. J Clin Oncol. 2010;28:1547-53.

8. Koizumi W, Narahara H, Hara T, Takagane A, Akiya T, Takagi M, et al. S-1 plus cisplatin versus S-1 alone for first-line treatment of advanced gastric cancer (SPIRITS trial): a phase III trial. Lancet Oncol. 2008;9:215-21.

9. Lee JL, Kang YK, Kang HJ, Lee KH, Zang DY, Ryoo BY, et al. A randomised multicentre phase II trial of capecitabine vs S-1 as first-line treatment in elderly patients with metastatic or recurrent unresectable gastric cancer. Br J Cancer. 2008;99:584-90.

10. Koizumi W, Akiya T, Sato A, Sakuyama T, Sasaki E, Tomidokoro T, et al. Phase II study of S-1 as first-line treatment for elderly patients over 75 years of age with advanced gastric cancer: the 
Tokyo cooperative oncology group study. Cancer Chemother Pharmacol. 2010;65:1093-9.

11. Vanita N. Guidelines for chemotherapy and dosing modifications. In: Chu E, DeVita Jr VT, editors. Physicians' cancer chemotherapy drug manual. Sudbury: Jones and Bartlett; 2007. p. 388-9.

12. Lichtman SM, Wildiers H, Launay-Vacher V, Steer C, Chatelut E, Aapro M. International society of geriatric oncology (SIOG) recommendations for the adjustment of dosing in elderly cancer patients with renal insufficiency. Eur J Cancer. 2007;43:14-34.

13. The Japanese Society of Nephrology and Pharmacotherapy, Japan. Available from: https://www.jsnp.org/docs/JSNP-yakuzai_dosing_30.pdf. Accessed 02 May 2017.

14. Brookmeyer R, Crowley J. A confidence interval for the median survival time. Biometrics. 1982;38:29-41.

15. Al-Batran SE, Hartmann JT, Probst S, Schmalenberg H, Hollerbach S, Hofheinz R, et al. Phase III trial in metastatic gastroesophageal adenocarcinoma with fluorouracil, leucovorin plus either oxaliplatin or cisplatin: a study of the Arbeitsgemeinschaft Internistische Onkologie. J Clin Oncol. 2008;26:1435-42.

16. Cunningham D, Starling N, Rao S, Iveson T, Nicolson M, Coxon $\mathrm{F}$, et al. Capecitabine and oxaliplatin for advanced esophagogastric cancer. N Engl J Med. 2008;358:36-46.

17. Yamada Y, Higuchi K, Nishikawa K, Gotoh M, Fuse N, Sugimoto N, et al. Phase III study comparing oxaliplatin plus S-1 with cisplatin plus S-1 in chemotherapy-naïve patients with advanced gastric cancer. Ann Oncol. 2015;26:141-8.

18. Santini D, Graziano F, Catalano V, Di Seri M, Testa E, Baldelli AM, et al. Weekly oxaliplatin, 5-fluorouracil and folinic acid (OXALF) as first-line chemotherapy for elderly patients with advanced gastric cancer: results of a phase II trial. BMC Cancer. 2006;6:125. 